\title{
BMJ Open Endoscopic resection for non-polypoid dysplasia in inflammatory bowel disease: a systematic review protocol
}

\author{
Yuelun Zhang, ${ }^{\oplus 1,2}$ Wei Chen, ${ }^{1}$ Yi Zhao, ${ }^{\oplus}{ }^{1}$ Dong Wu ${ }^{1}$
}

\begin{abstract}
To cite: Zhang Y, Chen W, Zhao Y, et al. Endoscopic resection for non-polypoid dysplasia in inflammatory bowel disease: a systematic review protocol. BMJ Open 2019;9:e029383. doi:10.1136/ bmjopen-2019-029383

- Prepublication history and additional material for this paper are available online. To view these files, please visit the journal online (http://dx.doi. org/10.1136/bmjopen-2019029383).
\end{abstract}

YZ and WC contributed equally.

YZ and WC are joint first authors.

Received 24 January 2019

Revised 20 August 2019

Accepted 27 August 2019

\begin{abstract}
Introduction Non-polypoid low-grade dysplasia in inflammatory bowel disease is associated with a medium increased risk of colorectal cancer, while treatment recommendations remain controversial. We aim to evaluate the efficacy and safety of endoscopic treatment for non-polypoid dysplasia in patients with inflammatory bowel disease.

Methods and analysis Medline, Embase, Cochrane Library, Scopus, Web of Science and clinical trials registry from database inception to the search date will be used to retrieve eligible studies. Studies that report the curative resection rate or any of other secondary outcomes of endoscopic treatment in patients with non-polypoid dysplasia in inflammatory bowel disease will be included in the analysis. We will conduct quantitative synthesis if the eligible studies are homogeneous judging from clinical and methodological perspectives.
\end{abstract}

Ethics and dissemination Ethical approval for this study was waived by the Ethics Committee of Peking Union Medical College Hospital because there are no individual data involved in the analysis and all the combined results will be retrieved from study-level data. We plan to disseminate results through peer-reviewed journals or conference abstracts.

PROSPERO registration number CRD42019120413.

\section{INTRODUCTION}

Inflammatory bowel disease (IBD) is a chronic relapsing disease including ulcerative colitis (UC) and Crohn's disease (CD). The annual incidence of IBD is 37.0-39.4/100 000 personyears in Western countries and 11.3/10 000 person-years in the Asian area. ${ }^{1}$ Patients with long-term IBD have an increased risk of colorectal cancer (CRG), and most cases of CRC are believed to arise from dysplasia. ${ }^{2}$ Here, dysplasia refers to an unequivocal neoplastic alteration of the colonic epithelium without evidence of tissue invasion, which is characterised by specific cytological and/or architectural changes to the epithelium, and CRC refers to lesions that show histological evidence of invasion through the muscularis mucosa into the submucosa. ${ }^{3}$ Besides, colitis-associated dysplasia should be distinguished from sporadic neoplasm by
Strengths and limitations of this study

The planned quantitative synthesis addressing endoscopic resection for non-polypoid in inflammatory bowel disease will overcome the limited statistical power in previous original studies.

- There is no restriction on population, study design or publication characteristics, providing an overall evidence map for clinical practice.

- Limited evidence from randomised controlled trials may weaken the confidence of the study conclusion.

comprehensive judgement based on the site, morphology and histological feature of the lesion according to the European consensus. ${ }^{4}$ The cumulative incidence of neoplasia (sporadic adenoma, UC-associated dysplasia and CRC) in patients with long-standing UC was $4.1 \%$ at 10 years, $14.1 \%$ at 20 years, $28.0 \%$ at 30 years and $38.9 \%$ at 40 years, with a CRC risk of $0.1 \%, 2.9 \%, 6.7 \%$ and $10.0 \%$, respectively. ${ }^{5}$ The HR of developing CRC in IBD patients with dysplasia compared with IBD patients without dysplasia was 7.8 for low-grade dysplasia (LGD) and 33.1 for highgrade dysplasia (HGD). ${ }^{5}$ Therefore, timely surveillance and early treatment of precancerous lesions (dysplasia) are essential to prevent CRC in IBD.

The Surveillance for Colorectal Endoscopic Neoplasia Detection and Management in Inflammatory Bowel Disease Patients International Consensus Recommendations (SCENIC) consensus classified IBD-dysplasia into visible and non-visible lesions, with visible lesions further divided into polypoid dysplasia (protruding from the mucosa into the lumen $\geq 2.5 \mathrm{~mm}$ ) and non-polypoid dysplasia $(<2.5 \mathrm{~mm}$ or no protrusion above the mucosa). ${ }^{6}$ There is a strong association between HGD and CRC (synchronous $^{7}$ or metachronous ${ }^{5}$ ), justifying colectomy as a reasonable treatment for patients with IBD-HGD. With regard to LGD, polypoid LGD is believed to be an indication 
for endoscopic resection, due to technical feasibility and much lower risk of recurrence. Treatment recommendations for non-polypoid LGD, however, remain controversial, ${ }^{8}$ since non-polypoid LGD is associated with a medium risk (eg, between polypoid $\mathrm{LGD}^{9}$ and $\mathrm{HGD}^{5}$ ) to develop CRC, ${ }^{10}$ but requires much higher endoscopic skill to resect it.

Endoscopic resection techniques for non-polypoid LGD consist of endoscopic mucosal resection (EMR) and endoscopic submucosal dissection (ESD). The safety of endoscopic resection for polypoid LGD has been confirmed by meta-analysis with a postoperative CRC risk of as low as 5/1000 person-years. ${ }^{9}$ Data on CRC risk after resection of non-polypoid dysplasia in IBD are scarce. Submucosal fibrosis and the obscure margin of non-polypoid dysplasia in IBD are responsible for the technical difficulties in endoscopic resection. ${ }^{11}$ With the development of endoscopic techniques, several studies started to fill the gap on endoscopic resection in the management of non-polypoid dysplasia. ${ }^{12}$

The small sample sizes and heterogeneity of these studies compromised the reliability of their conclusions. Therefore, it is crucial to perform a systematic review collecting and evaluating available studies and to establish a body of evidence for IBD patients with non-polypoid dysplasia undergoing endoscopic resection.

\section{Objectives}

This research protocol aims to evaluate the efficacy (curative resection rate, for example) and safety (such as recurrence, bleeding and perforation) of endoscopic treatment for non-polypoid dysplasia in patients with IBD.

\section{METHODS AND ANALYSIS}

The protocol was registered on the PROSPERO (International Prospective Register of Systematic Reviews) website and reported in compliance with the Preferred Reporting Items for Systematic Review and Meta-Analysis Protocols statement. ${ }^{13}$ Any further amendments in the protocol and conduct of this systematic review will be recorded and submitted to the PROSPERO website and reported in future publications.

\section{Inclusion criteria for study selection \\ Types of studies}

Eligible studies may include retrospective or prospective cohort studies (single-arm or multiple exposure groups), consecutive case series, cross-sectional studies, or randomised controlled trials that reported at least one of the primary outcomes (curative resection rate) and secondary outcomes (en-bloc resection rate, CRC incidence rate, local recurrence rate, metachronous recurrence rate, rate of postoperative bleeding and perforation during the procedure, rate of submucosal fibrosis, and overall survival).

\section{Types of participants}

Patients diagnosed with IBD and non-polypoid dysplasia should be confirmed by clinical, endoscopic and histological evaluation. Here, dysplasia refers to an unequivocal neoplastic alteration of the colonic epithelium without evidence of tissue invasion, which is characterised by specific cytological and/or architectural changes to the epithelium. ${ }^{3}$ Due to the update of terminology, ${ }^{6}$ the term non-polypoid dysplasia here includes flat dysplasia, Paris 0 -II lesions and laterally spreading tumours (lesions reach a large $(>10 \mathrm{~mm})$ lateral diameter without increasing their height or protrusion above the mucosa) ${ }^{6}{ }^{614}$ To avoid missing eligible studies, we will carefully check the definition of dysplasia-associated lesion or mass (DALM) and will only include those that fulfil the criteria for non-polypoid dysplasia, since the term DALM is confusing and is used to describe all irregular, diffuse masses or plaque lesions in actively or previously inflamed areas of the colon.

\section{Types of interventions}

Endoscopic resection includes EMR and ESD for non-polypoid dysplasia in IBD.

\section{Types of outcome measures}

The primary outcome in our systematic review is curative resection rate ( $\mathrm{R} 0$ resection with submucosal invasion $<1000 \mathrm{~mm}$, absent lymphovascular involvement) of non-polypoid dysplasia. ${ }^{15}$ The secondary outcomes in this systematic review include en-bloc resection rate, $\mathrm{R} 0$ resection rate (en-bloc resection with negative horizontal and vertical margin), CRC incidence rate, local recurrence rate, metachronous recurrence rate, rate of postoperative bleeding and perforation during the procedure, rate of submucosal fibrosis, and overall survival.

\section{Literature search for identification of studies}

Potentially relevant studies will be searched using Medline, Embase, Cochrane Controlled Register of Trials, Scopus, Web of Science and ClinicalTrials.gov registry from database inception up to 1 July 2019. Free text and medical subject heading terms relevant to endoscopy, inflammatory bowel disease and dysplasia will be used in the literature search. We will not use any filter for study design. Hand search of the bibliographies of relevant review and systematic review articles will be also conducted. We will set no language limitation in the literature search. The detailed literature search strategy is shown in online supplementary table S1.

\section{Data collection and analysis}

\section{Selection of studies}

Records retrieved from the literature search will be imported into EndNote, and duplicate citations will be removed. Two investigators (WC and $\mathrm{YZ}$ ) will independently assess the eligibility of the studies by reading the title and the abstract, and the full texts of potentially eligible studies will be used to determine the final eligibility. Disagreement during the literature screening and 
inclusion process will be resolved by discussion with a methodologist (YLZ) and a gastroenterologist (DW). In each stage, we will record reasons for excluding citation in the EndNote library.

\section{Data extraction and management}

Data will be extracted into an Excel extraction form by one investigator (WC) and double-checked by one methodologist (YLZ). We will retrieve the following information from each eligible study:

- Basic information of the study: author, publication year, design and sample size.

- Patient characteristics: age, sex, duration of disease, inflammatory endoscopic/histological activity, lesion size, lesion location, submucosal fibrosis, different types of IBD (UC and CD) and primary sclerosing cholangitis.

- Detailed information on the endoscopic equipment for surveillance and techniques for therapy: whitelight endoscopy (WLE), chromoendoscopy (CE), narrow band imaging (NBI), endoscopic mucosal resection (EMR), endoscopic submucosal disection (ESD) and so on.

- Outcome data: number of patients with en-bloc/R0/ curative resection, postoperative bleeding and perforation, submucosal fibrosis, CRC incidence, local recurrence, metachronous recurrence, and overall survival in long-term follow-up.

We will make the most extensive use of all the available materials of the relevant studies, including but not limited to the publications, unpublished reports, information from study registries and online appendices. If the vital information is unavailable in the above sources, we will try to contact the investigators to get the relevant data through email. We will transform all the extracted data into the international system of units.

\section{Risk of bias assessment}

If relevant evidence is available, we will use the Cochrane Collaboration's tool to assess the risk of bias in randomised controlled trials and the Newcastle-Ottawa Scale to evaluate the risk of bias in two-armed cohort studies. For single-arm cohort studies, we will use a modified tool to assess the risk of bias of eligible studies based on the Agency for Healthcare Research and Quality (AHRQ) tool. ${ }^{16}$ The risk of bias will be evaluated by one investigator (WC) and double-checked by one methodologist (YLZ). Any disagreement will be resolved by discussion with a senior investigator (DW). Detailed criteria of the modified AHRQ tool are shown in online supplementary table S2.

\section{Statistical analysis}

We will first describe the basic characteristics and the risk of bias of eligible studies. If eligible studies are in different designs, they will be reported and synthesised separately. We will assess the eligible studies in terms of heterogeneity by evaluating the clinical and methodological differences qualitatively, and if there is significant heterogeneity quantitative synthesis will be abandoned.

This planned systematic review aims to collect evidence from randomised clinical trials and observational studies. However, we anticipate that the data on outcomes of interest will be mostly reported in single-arm cohort studies, lacking comparison between randomly allocated intervention groups. Considering the potential clinical and methodological heterogeneity among eligible observational studies, we will use a random-effects model to combine the effect. ${ }^{17}$ The curative resection rate and all the secondary outcomes with $95 \%$ CI will be pooled as proportion with logit transformation if there are enough data supporting the synthesis. ${ }^{18}$ Clopper-Pearson interval method will serve to estimate the 95\% CI in each study. ${ }^{19}$

Between-study variance will be estimated using the restricted maximum likelihood estimator. ${ }^{20}$ We will measure heterogeneity between studies using $\mathrm{I}^{2}$ statistics, and an $\mathrm{I}^{2}$ value larger than $50 \%$ will be defined as substantial heterogeneity. ${ }^{21}$

We do not plan to assess reporting bias in this systematic review since the hypothesis behind the commonly applied methods for detecting reporting bias may not apply to single-arm rates or proportions. ${ }^{22}$

Subgroup analysis will be conducted with regard to lesion size, lesion location, duration of the disease, submucosal fibrosis and different types of IBD (UC and $\mathrm{CD}$ ). We will perform post-hoc subgroup analysis if there is evidence that some crucial sources contribute to the statistical heterogeneity. The potential sources of heterogeneity will be further assessed using multiple random-effects meta-regression to explore the independent contribution of each variable to the main outcome. Results from post-hoc subgroup analysis will be interpreted as hypothesis-generating rather than definite evidence for subgroup difference.

Sensitivity analysis using different transformation methods (log transformation, Freeman-Tukey double arcsine transformation, arcsine transformation or raw proportion without transformation) will be conducted to check if the main findings are robust. All statistical analyses will be completed in R V.3.5.2 (R Foundation for Statistical Computing, Vienna, Austria) with a two-sided $\alpha$ of 0.05 .

\section{Grading the quality of evidence}

The quality of evidence for all the outcomes will be assessed using the Grading of Recommendations Assessment, Development and Evaluation (GRADE) working group methodology. ${ }^{23}$ Detailed evaluation methods will follow the recommendations from GRADE working group.

\section{Patient and public involvement}

Patients or the public are not involved in the design and conception of this study. 


\section{Ethics and dissemination}

This is a research protocol for a systematic review and the data are not collected yet; hence, there are no data published in a data repository. The results will be disseminated through peer-reviewed publications or conference abstracts.

\section{DISCUSSION}

Indefinite margins and submucosal fibrosis add to the technical difficulties for endoscopic resection of non-polypoid dysplasia. Our meta-analysis will evaluate the overall en-bloc/ $\mathrm{R} 0 /$ curative resection rate and implement subgroup analysis according to potential influencing factors such as lesion size and inflammatory activity to select patients who may benefit most from endoscopic therapy. Given that the incidence of metachronous dysplasia and CRC remains largely unknown in non-polypoid dysplasia after endoscopic resection, ${ }^{6}$ this planned systematic review and meta-analysis will provide useful information on long-term prognosis. We will also compare our results with the evidence from polypoid dysplasia which was cited by $\mathrm{ECCO}^{8}$ and SCENIC 6 guidelines, which may help clinicians make reasonable therapeutic strategies for the management of non-polypoid dysplasia in IBD. Besides, endoscopic resection has the advantage of less complication risk and patient preference ${ }^{24}$; therefore, if endoscopic resection proves reasonably effective and safe for the management of non-polypoid dysplasia, it may become the first-choice therapy in such patients. However, this systematic review have some potential limitations. The best evidence evaluating the effect of endoscopic resection should come from randomised controlled trials comparing endoscopic resection versus other therapies in patients with non-polypoid dysplasia in IBD. However, based on our pilot literature search, few studies, if any, have addressed this problem in a randomised design. The data synthesis from single-arm cohort studies or other relevant data sources may be highly sensitive to the selection of population and the practice setting. Hence, we are justified to expect significant heterogeneity across studies. Moreover, the potentially limited follow-up periods may be insufficient to observe longterm outcome events such as CRC incidence, local recurrence and overall survival. The underlying heterogeneity regarding clinical and methodological considerations should be evaluated using subgroup analysis or meta-regression. Nevertheless, the number of eligible studies is expected to be small, given the relatively late application of endoscopic techniques in practice, limiting our ability to analyse influencing factors for treatment effectiveness.

Acknowledgements We thank Dr Yang Chen (Department of Gastroenterology, Peking Union Medical College Hospital, Chinese Academy of Medical Sciences and Peking Union Medical College) and Dr Wen Shi (Department of Gastroenterology, Peking Union Medical College Hospital, Chinese Academy of Medical Sciences and
Peking Union Medical College) for providing critical comments on the overall design and the manuscript.

Contributors DW is the guarantor of this systematic review and launched this research. WC and YZ completed the pilot literature search and will conduct the formal literature search and screening. YLZ designed the data extraction form, the tool for risk of bias assessment and the data synthesis plan. WC and YZ will extract the data. YLZ will conduct the quantitative synthesis. DW, WC, YZ and YLZ will interpret the results. All the authors contributed to the drafting of the manuscript and approved the publication.

Funding This systematic review and meta-analysis is funded by Peking Union Medical College (10023201700105). The sponsor has no role in study design, data collection, data analysis and result interpretation.

Competing interests None declared.

Patient consent for publication Not required.

Ethics approval Formal ethical approval is waivered since there are no individual data involved in the analysis and all the combined results will be retrieved from study-level data.

Provenance and peer review Not commissioned; externally peer reviewed.

Open access This is an open access article distributed in accordance with the Creative Commons Attribution Non Commercial (CC BY-NC 4.0) license, which permits others to distribute, remix, adapt, build upon this work non-commercially, and license their derivative works on different terms, provided the original work is properly cited, appropriate credit is given, any changes made indicated, and the use is non-commercial. See: http://creativecommons.org/licenses/by-nc/4.0/.

\section{REFERENCES}

1. Molodecky NA, Soon IS, Rabi DM, et al. Increasing incidence and prevalence of the inflammatory bowel diseases with time, based on systematic review. Gastroenterology 2012;142:46-54.

2. Leidenius M, Kellokumpu I, Husa A, et al. Dysplasia and carcinoma in longstanding ulcerative colitis: an endoscopic and histological surveillance programme. Gut 1991;32:1521-5.

3. Bosman FT, Carneiro F, Hruban RH, et al. WHO classification of tumours of the digestive system. World Health Organization, 2010.

4. Magro F, Langner C, Driessen A, et al. European consensus on the histopathology of inflammatory bowel disease. J Crohns Colitis 2013;7:827-51.

5. Choi C-HR, Rutter MD, Askari A, et al. Forty-Year analysis of colonoscopic surveillance program for neoplasia in ulcerative colitis: an updated overview. Am J Gastroenterol 2015;110:1022-34.

6. Laine L, Kaltenbach T, Barkun A, et al. Scenic international consensus statement on surveillance and management of dysplasia in inflammatory bowel disease. Gastroenterology 2015;148:639-51.

7. Shanahan F, Weinstein WM, Bernstein CN. Are we telling patients the truth about surveillance colonoscopy in ulcerative colitis? The Lancet 1994;343:71-4.

8. Van Assche G, Dignass A, Bokemeyer B, et al. Second European evidence-based consensus on the diagnosis and management of ulcerative colitis Part 3: special situations. J Crohn's Colitis 2013;7:1-33.

9. Wanders LK, Dekker E, Pullens B, et al. Cancer risk after resection of polypoid dysplasia in patients with longstanding ulcerative colitis: a meta-analysis. Clin Gastroenterol Hepatol 2014;12:756-64.

10. Thomas T, Abrams KA, Robinson RJ, et al. Meta-Analysis: cancer risk of low-grade dysplasia in chronic ulcerative colitis. Aliment Pharmacol Ther 2007;25:657-68.

11. East JE, Toyonaga T, Suzuki N. Endoscopic management of nonpolypoid colorectal lesions in colonic IBD. Gastrointest Endosc Clin N Am 2014;24:435-45.

12. Soetikno R, East J, Suzuki N, et al. Endoscopic submucosal dissection for nonpolypoid colorectal dysplasia in patients with inflammatory bowel disease: in medias Res. Gastrointest Endosc 2018;87:1085-94.

13. Shamseer L, Moher D, Clarke M, et al. Preferred reporting items for systematic review and meta-analysis protocols (PRISMA-P) 2015: elaboration and explanation. BMJ 2015;349:g7647.

14. Anon. The Paris endoscopic classification of superficial neoplastic lesions: esophagus, stomach, and colon: November 30 to December 1, 2002. Gastrointest Endosc 2003;58(6 Suppl):S3-43.

15. lacopini $F$, Saito $Y$, Yamada M, et al. Curative endoscopic submucosal dissection of large nonpolypoid superficial neoplasms 
in ulcerative colitis (with videos). Gastrointest Endosc 2015;82:734-8.

16. Viswanathan M, Ansari MT, Berkman ND, et al. AHRQ methods for effective health care: assessing the risk of bias of individual studies in systematic reviews of health care interventions. In: Methods guide for effectiveness and comparative effectiveness reviews. Rockville (MD): Agency for Healthcare Research and Quality (US), 2008.

17. Mueller M, D'Addario M, Egger M, et al. Methods to systematically review and meta-analyse observational studies: a systematic scoping review of recommendations. BMC Med Res Methodol 2018:18:1-18.

18. Barendregt JJ, Doi SA, Lee YY, et al. Meta-Analysis of prevalence. $J$ Epidemiol Community Health 2013;67:974-8.

19. Clopper CJ, Pearson ES. The use of confidence or fiducial limits illustrated in the case of the binomial. Biometrika 1934;26:404-13.
20. Munn Z, Moola S, Lisy K, et al. Methodological guidance for systematic reviews of observational epidemiological studies reporting prevalence and cumulative incidence data. Int J Evid Based Healthc 2015;13:147-53.

21. Higgins JPT, Green S. Cochrane Handbook for systematic reviews of interventions. Cochrane Collab, 2011.

22. Hunter JP, Saratzis A, Sutton AJ, et al. In meta-analyses of proportion studies, funnel plots were found to be an inaccurate method of assessing publication bias. J Clin Epidemiol 2014;67:897-903.

23. Guyatt GH, Oxman AD, Vist GE, et al. Grade: an emerging consensus on rating quality of evidence and strength of recommendations. BMJ 2008;336:924-6.

24. Siegel CA, Schwartz LM, Woloshin S, et al. When should ulcerative colitis patients undergo colectomy for dysplasia? mismatch between patient preferences and physician recommendations. Inflamm Bowel Dis 2010;16:1658-62. 